\title{
NOTE ON THE CLASS NUMBER OF REAL QUADRATIC FIELDS
}

\section{CARLITZ}

1. Let $p$ be a prime $\equiv 1(\bmod 4)$ and let $h(p)$ denote the class number of the real quadratic field $R\left(p^{1 / 2}\right)$; let $\epsilon=\left(t+u p^{1 / 2}\right) / 2$ denote the fundamental unit of the field $(\epsilon>1)$. Ankeny, Artin, and Chowla [1] have stated the following results, as well as certain more general ones.

$$
\begin{aligned}
& 4 u h / t \equiv-\sum_{r=1}^{(p-1) / 4} \frac{1}{r}\left(\frac{r}{p}\right)(\bmod p) \quad(p \equiv 5(\bmod 8)) ; \\
& u h / t \equiv B_{(p-1) / 2}(\bmod p),
\end{aligned}
$$

where $B_{m}$ denotes a Bernoulli number in the even suffix notation;

$$
2 u h / t \equiv(A+B) / p(\bmod p),
$$

where $A$ is the product of the quadratic residue of $p$ between 0 and $p$, and $B$ is the product of the nonresidues of $p$ between 0 and $p$. Proofs of these results (except (1.3)) appear in [2].

In this note we wish to point out that if we assume (1.2), then (1.1) and (1.3) can be proved quite simply. We remark that for $p \equiv 1(\bmod 8)$ the right member of $(1.1)$ is congruent to $0(\bmod p)$.

2. To show that (1.2) implies (1.1) we have

$$
\begin{aligned}
S=\sum_{r=1}^{(p-1) / 4} \frac{1}{r}\left(\frac{r}{p}\right) & \equiv \sum_{1}^{(p-1) / 4} r^{(p-3) / 2} \\
& \equiv \frac{B_{(p-1) / 2}(3 / 4)-B_{(p-1) / 2}}{(p-1) / 2}(\bmod p),
\end{aligned}
$$

where $B_{m}(x)$ is the Bernoulli polynomial of degree $m$. Since $[4$, p. 22]

$$
B_{2 m}(3 / 4)=B_{2 m}(1 / 4)=\left(2^{1-4 m}-2^{-2 m}\right) B_{2 m},
$$

we see that (2.1) becomes

$$
S \equiv-2\left(1-2^{(p-1) / 2)} B_{(p-1) / 2} \equiv \begin{cases}0 & p \equiv 1(\bmod 8) \\ -4 B_{(p-1) / 2} & p \equiv 5(\bmod 8) .\end{cases}\right.
$$

This evidently proves (1.1).

The question is raised in $[2$, p. 480$]$ whether $u$ can be divisible by $p$. According to $(1.2)$ this can only happen if $B_{(p-1) / 2} \equiv 0(\bmod p)$.

Received by the editors December 6, 1952. 
More precisely $B_{(p-1) / 2} \equiv 0$ if and only if either $h \equiv 0$ or $u \equiv 0$. In this connection it is of interest to note that for $p \equiv 3(\bmod 4), B_{(p+1) / 2}$ $\not \equiv 0(\bmod p)$. This is a consequence of the well known formula $(p>3)$

$$
\begin{aligned}
H(p) & =\frac{1}{2-(2 / p)} \sum_{r=1}^{(p-1) / 2}\left(\frac{r}{p}\right) \\
& \equiv \frac{2}{2-(2 / p)}\left\{B_{(p-1) / 2}(1 / 2)-B_{(p+1) / 2}\right\} \\
& \equiv-2 B_{(p+1) / 2}(\bmod p) ;
\end{aligned}
$$

here $H(p)$ denotes the class number of the imaginary quadratic field $R\left((-p)^{1 / 2}\right)$; clearly $H(p)<p$.

3. To prove (1.3) we follow Nielsen [3, Chapter 20]. Put

$$
\prod_{s=1}^{(p-1) / 2} a_{s}=-1+p \Omega_{p}, \quad \prod_{s=1}^{(p-1) / 2} b_{s}=1-p \Omega_{p}^{\prime},
$$

where the $a_{0}$ denote the quadratic residues in the interval $0, p$, and the $b$, denote the non residues. Thus, in the notation of (1.3), we have

$$
A+B=p\left(\Omega_{p}-\Omega_{p}^{\prime}\right) .
$$

Now if we put

$$
r^{p-1}=1+p k(r)
$$

then it follows from (3.1) that

$$
\Omega_{p} \equiv \sum_{s=1}^{(p-1) / 2} k\left(a_{s}\right), \quad \Omega_{p}^{\prime} \equiv \sum_{s=1}^{(p-1) / 2} k\left(b_{s}\right)(\bmod p) .
$$

Consequently

$$
\Omega_{p}-\Omega_{p}^{\prime} \equiv \frac{1}{p} \sum_{r=1}^{p-1}\left(\frac{r}{p}\right)\left(r^{p-1}-1\right)(\bmod p),
$$

so that

$$
\begin{aligned}
p\left(\Omega_{p}-\Omega_{p}^{\prime}\right) & \equiv \sum_{1}^{p-1}\left(r^{8(p-1) / 2}-r^{(p-1) / 2}\right) \\
& \equiv \frac{B_{(3 p-1) / 2}(p)-B_{(3 p-1) / 2}}{(3 p-1) / 2}-\frac{B_{(p+1) / 2}(p)-B_{(p+1) / 2}}{(p+1) / 2} \\
& \equiv p\left(B_{3(p-1) / 2}-B_{(p-1) / 2}\right)\left(\bmod p^{2}\right),
\end{aligned}
$$

and 


$$
\Omega_{p}-\Omega_{p}^{\prime} \equiv B_{3(p-1) / 2}-B_{(p-1) / 2}(\bmod p) .
$$

Since by Kummer's congruence [3, Chapter 14]

$$
\frac{B_{3(p-1) / 2}}{3(p-1) / 2} \equiv \frac{B_{(p-1) / 2}}{(p-1) / 2}(\bmod p)
$$

it is clear that (3.3) reduces to

$$
\Omega_{p}-\Omega_{p}^{\prime} \equiv 2 B_{(p-1) / 2}(\bmod p) .
$$

Hence (3.2) implies

$$
\frac{1}{p}(A+B) \equiv 2 B_{(p-1) / 2}
$$

which evidently proves (1.3).

\section{REFERENCES}

1. N. C. Ankeny, E. Artin, and S. Chowla, The class-number of real quadratic fields, Proc. Nat. Acad. Sci. U.S.A. vol. 37 (1951) pp. 524-525.

2. - The class-number of real quadratic fields, Ann. of Math. (2) vol. 56 (1952) pp. $479-493$.

3. N. Nielsen, Traité elementaire des nombres de Bernoulli, Paris, 1923.

4. N. E. Nörlund, Vorlesungen uber Differenzenrechnung, Berlin, 1924.

DUKe UNIVERSITY 